\title{
Erratum: Capacitively coupled arrays of multiplexed flexible silicon transistors for long-term cardiac electrophysiology
}

Hui Fang, Ki Jun Yu, Christopher Gloschat, Zijian Yang, Enming Song, Chia-Han Chiang, Jianing Zhao, Sang Min Won, Siyi Xu, Michael Trumpis, Yiding Zhong, Seung Won Han, Yeguang Xue, Dong Xu, Seo Woo Choi, Gert Cauwenberghs, Matthew Kay, Yonggang Huang, Jonathan Viventi, Igor R. Efimov and John A. Rogers

Nature Biomedical Engineering 1, 0038 (2017); published 1 March 2017; corrected 9 March 2017.

In the version of this Article originally published, the single-voltage trace in Fig. $3 \mathrm{~b}$ did not display correctly. This has now been corrected. 